\title{
Vector Absorbing Boundary Conditions for Nodal or Mixed Finite Elements
}

\author{
J.L. Yao Bi, L. Nicolas, A. Nicolas \\ CEGELY - URA CNRS 829 \\ BP163 - 69131 Ecully cedex - France
}

\begin{abstract}
We present 2D and 3D vector absorbing boundary conditions for unbounded microwave problems. Coupling with vector formulation leads to a non-symmetric system matrix. We show how it is possible to symmetrise these conditions with the same degree of precision. Edge and corner conditions are also presented for rectangular boundaries.
\end{abstract}

\section{INTRODUCTION}

Modeling an electromagnetic problem with a finite element (F.E.) method requires the truncation of the infinite domain with a fictitious boundary. Absorbing boundary conditions (A.B.C.) taking into account the external domain are then written on this outer boundary. In their asymptotic form, when the boundary is located at the infinite, these conditions have to be identified with the Sommerfeld (for scalar wave equation) or the Silver-Müller (for vector wave equation) radiation conditions.

These boundary conditions are generally presented as integral or differential operators [1]. They can be of two types: global or local. Global operators are exact and allow in principle to locate the outer boundary as close as possible to the modeled device. But the resulting boundary matrix is full and non-symmetric, preventing their use in 3D. On an other hand, local A.B.C. preserve the sparsity of the F.E. system matrix, but they are built up as an approximation of the exact solution: they are not totally absorbing and spurious reflexions come back inside the F.E. domain. Because of the use in 3D, we will consider here only local A.B.C.

One can find many kinds of local A.B.C., but two of them are often shown up: the Engquist-Majda (E.M.) condition [2], based on the approximation of a non-local pseudodifferential operator by a local differential operator, absorbs perfectly plane waves at given incidence angles. The Bayliss-Turkel (B.T.) condition [3] is based on an asymptotic expansion of the solution of the scalar wave equation; it is built as a sequence of differential operators which annihilate the first terms of this expansion.

The location of the A.B.C. is a crucial point, especially in 3D: its location as close as possible to the microwave device saves CPU time and memory space. Depending on the geometry of the device, one will choose either E.M. type of

Manuscript received July 10, 1995.

L. Nicolas, e-mail laurent@trotek.ec-lyon.fr, tel (33) 721861 16, fax (33) 784337 17; A. Nicolas, e-mail nicolas@trotek.ec-lyon.fr, tel (33) 72 186096 , fax (33) 78433717 .
A.B.C., working with a rectangular outer boundary, or B.T. type, working with a circular boundary.

The object of this paper is to review both of these A.B.C., to present their vector form and to show how they can be symmetrized. A special attention is paid to their theoretical accuracy. For the case of the E.M. A.B.C., we also show how edge and corner problems may be solved.

\section{2D BAYLISS-TURKEL A.B.C.}

\section{A. 2 D scalar Bayliss-Turkel A.B.C.}

A solution to the $2 \mathrm{D}$ cylindrical scalar wave equation may be developed as the asymptotic expansion [4]:

$\psi(r, \theta)=\frac{e^{-j k r}}{r^{1 / 2}} \sum_{n=0}^{\infty} \frac{a_{n}(\theta)}{r^{n}}$

where $\psi$ is the magnetic or electric field.

The basic idea of Bayliss and Turkel was to build a sequence of differential operators to cancel out the first terms of (1). The sequence of B.T. A.B.C. for the 2D scalar wave equation is then given recursively by [3]:

$\mathrm{Bm} \psi=\mathrm{O}\left(\mathrm{R}^{-2 \mathrm{~m}-1 / 2}\right)$ with $\mathrm{B}_{\mathrm{m}}=\prod_{\mathrm{p}=1}^{\mathrm{m}}\left(\frac{\partial}{\partial \mathrm{r}}+\mathrm{jk}+\frac{4 \mathrm{p}-3}{2 \mathrm{R}}\right)$

To minimize the error due to the operator Bm, either one can move away the A.B.C., or one can use an higher order condition. A larger radius R leads to a larger F.E. domain and then to a larger system matrix. On an other hand, the numerical implementation of high order conditions $(m \geq 2)$ implies numerical derivations of high order, which lead to the use of high order finite elements. Furthermore, because of the asymptotic nature of the $\mathrm{Bm}$ operators, it does not lead necessarily to a continuous improvement of the solution when the boundary come close to the microwave objects [5]. Actually, $2^{\text {nd }}$ order conditions are often sufficient to locate the structure close to the device (Fig. 2).

To be coupled with a finite element formulation, radial derivatives are replaced with angular derivatives. After expansion of the coefficients, second order B.T. condition is given by (3), similar to the condition found in [5]: 
$\frac{\partial \psi}{\partial \mathrm{r}}=\mathrm{a} \psi+\mathrm{b} \frac{\partial^{2} \psi}{\partial \theta^{2}}+\mathrm{O}\left(\mathrm{R}^{-9 / 2}\right)$

where $a(R)=-j k-\frac{1}{2 R}-\frac{1}{8 k^{2}}+\frac{1}{8 k^{2} R^{3}}$

$$
b(R)=-\frac{j}{2 k R^{2}}+\frac{1}{2 k^{2} R^{3}}
$$

\section{B. $2 D$ vector Bayliss-Turkel A.B.C.}

The scalar A.B.C. is not directly suitable to a mixed-based vector finite element formulation [6], because there is no link between the components of the field in the scalar condition. To obtain a vector form of this A.B.C., we just have to state that each cartesian component of the field $\mathbf{A}$ verifies the previous scalar condition. The vector field $\mathbf{A}$ verifies then the condition:

$\frac{\partial \mathbf{A}}{\partial \mathrm{r}}=\mathrm{a} \mathbf{A}+\mathrm{b} \frac{\partial^{2} \mathbf{A}}{\partial \theta^{2}}$

Using the divergence-free condition for the vector $\mathbf{A}$ and basis relations in cylindrical coordinates, we get:

$\frac{\partial \mathbf{A}_{t}}{\partial \mathrm{r}}=(\mathrm{a}-\mathrm{b}) \mathbf{A}_{\mathrm{t}}+\mathrm{b} \frac{\partial^{2} \mathbf{A}_{\mathrm{t}}}{\partial \theta^{2}}+2 \operatorname{Rb} \nabla_{\mathrm{t}}(\mathbf{r} \cdot \mathbf{A})$

with $\mathbf{A}_{\mathrm{t}}=\mathrm{A}_{\theta} \mathbf{e}_{\theta}$ and $\nabla_{\mathrm{t}}=\frac{1}{\mathrm{r}} \frac{\partial}{\partial \theta} \mathbf{e}_{\theta}$

Using vector identities, (5) may be rewritten as the $2 \mathrm{D}$ vector B.T. absorbing condition [7]:

$\mathbf{r} \times \nabla \times \mathbf{A}=\mathrm{a}^{\prime} \mathbf{A}_{\mathrm{t}}+\mathrm{b}^{\prime} \nabla_{\mathrm{t}}\left(\nabla \cdot \mathbf{A}_{\mathrm{t}}\right)+\mathrm{g} \nabla_{\mathrm{t}}(\mathbf{r} \cdot \mathbf{A})+\mathrm{O}\left(\mathrm{R}^{-9 / 2}\right)$

where $a^{\prime}(R)=j k-\frac{1}{2 R}-\frac{3 j}{8 k R^{2}}+\frac{3}{8 k^{2} R^{3}}+O\left(R^{-4}\right)$

$$
\begin{aligned}
& b^{\prime}(R)=\frac{j}{2 k}-\frac{1}{2 k^{2} R}+O\left(R^{-2}\right) \\
& g(R)=1+\frac{j}{k R}-\frac{1}{k^{2} R^{2}}+O\left(R^{-3}\right)
\end{aligned}
$$

When inserted in the F.E. formulation, (6) leads to a nonsymmetric system matrix, due to the last $\nabla_{\mathrm{t}}$ term: hence it can be seen as a non-symmetric condition.

\section{C. $2 D$ symmetric vector Bayliss-Turkel A.B.C.}

It is however important to symmetrize the previous condition for storage capabilities considerations. A first method, suggested in [8] for $3 \mathrm{D}$, substitutes the first derivative of the radial component of $\mathbf{A}$ in (6) by the derivatives of the tangential component of $\mathbf{A}$, by using the A.B.C. itself for the r.A term. But the residual error goes from $O\left(\mathrm{r}^{-9 / 2}\right)$ to $\mathrm{O}\left(\mathrm{r}^{-7 / 2}\right)$, and it can be seen as a weakening of the condition. A better expression for r.A, obtained from (4), is given by [7]: $\mathbf{r} \cdot \mathbf{A}=-\frac{\mathrm{j}}{\mathrm{k}}\left(1+\frac{\mathrm{j}}{2 \mathrm{kR}}\right) \nabla \cdot \mathbf{A t}+\mathrm{O}\left(\mathrm{R}^{-7 / 2}\right)$

The Substitution in (6) leads to the following symmetric absorbing boundary condition, which has theoritically the same precision as the non-symmetric one:

$\mathbf{r} \times \nabla \times \mathbf{A}=\mathrm{a}^{\prime \prime} \mathbf{A}_{\mathbf{t}}+\mathrm{b}^{\prime \prime} \nabla_{\mathrm{t}}(\nabla . \mathbf{A} \mathbf{t})+\mathrm{O}\left(\mathrm{R}^{-9 / 2}\right)$

where $a^{\prime \prime}(R)=j k-\frac{1}{2 R}-\frac{3 j}{8 k R^{2}}+\frac{3}{8 k^{2} R^{3}}+O\left(R^{-4}\right)$

$$
b^{\prime \prime}(R)=-\frac{j}{2 k}+\frac{1}{k^{2} R}+O\left(R^{-2}\right)
$$

Note: this derivation of symmetric and non-symmetric conditions may be easily extended to the $3 \mathrm{D}$ case.

\section{Numerical example.}

As a numerical example, we modelize the scattering of a $\mathrm{TE}^{\mathrm{Z}}$ wave by a perfect electric conducting cylinder. An analytical solution may be found in [9]. We compare here the accuracies given by an exact A.B.C. (B.E.M.) and the Bayliss-Turkel condition (scalar and symmetric vector), when the outer boundary is moved away from the cylinder (from $0.25 \lambda$ to $3 \lambda$ ). The mesh density is about 20 nodes per wavelength; $\mathrm{kr}=\pi$, where $\mathrm{r}$ is the radius of the cylinder.

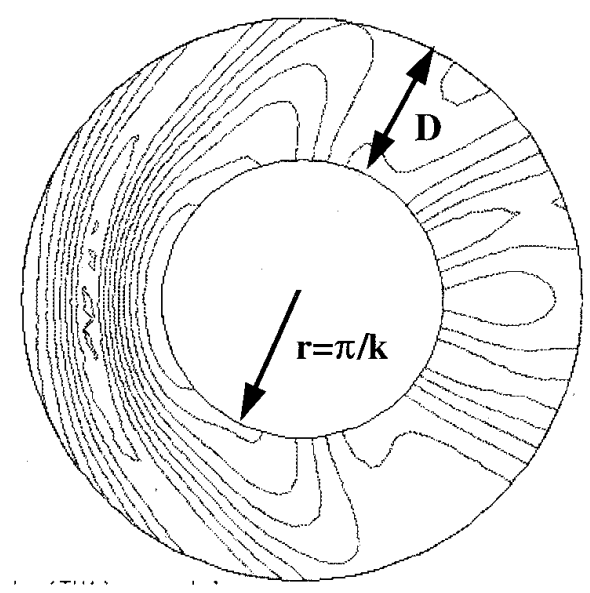

Fig. 1. Scattering by a p.e.c. cylinder Magnitude of the magnetic field $\mathrm{Hz}$.

Fig. 2 shows the global error, estimated as in [10]. This error becomes lower than $2 \%$ when the distance between the cylinder and the outer boundary is greater than $0.75 \lambda$. At $1 \lambda$, results may be considered as good. Note that, for a distance $D$ greater than $1.5 \lambda$, it was not possible to use the B.E.M., because of the CPU time and the memory storage. 


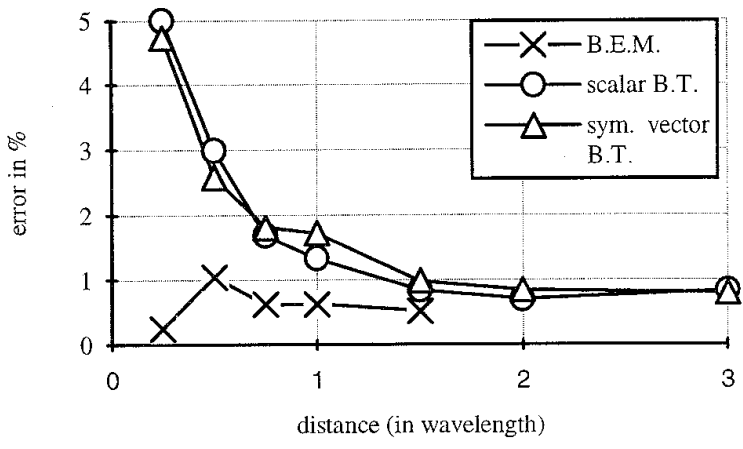

Fig. 1. Scattering by a p.e.c. cylinder: global error as a function of the distance between the cylinder and the outer boundary.

\section{2D ENGQUIST-MAJDA A.B.C}

If the geometry of the device is not adapted to a $2 \mathrm{D}$ circular or a 3D spherical outer boundary, it would be better to use an Engquist-Majda condition, which is working with a rectangular outer boundary, in order to minimize the number of nodes of the mesh generated in the free space.

\section{A. $2 D$ scalar Engquist-Majda A.B.C.}

The two-dimensional wave equation is written in an operator form, which is itself factored into a forward operator $\mathrm{L}_{\mathrm{f}}$ and a backward operator $\mathrm{L}_{\mathrm{b}}$ :

$$
\begin{gathered}
\mathrm{L} \psi=\mathrm{L}_{\mathrm{b}} \mathrm{L}_{\mathrm{f}} \psi=0, \text { with } \mathrm{L}_{\mathrm{f}}=\frac{\partial}{\partial \mathrm{x}}+\frac{1}{\mathrm{c}} \frac{\partial}{\partial \mathrm{t}} \sqrt{1-\mathrm{s}^{2}}, \\
\mathrm{~s}=\frac{\partial / \partial \mathrm{y}}{\partial / \partial \mathrm{t}}, \quad \mathrm{L}_{\mathrm{b}}=\frac{\partial}{\partial \mathrm{x}}-\frac{1}{\mathrm{c}} \frac{\partial}{\partial \mathrm{t}} \sqrt{1-\mathrm{s}^{2}}
\end{gathered}
$$

Engquist and Majda showed that the $\mathrm{L}_{b}$ operator applied to $\psi$ will absorb a backward wave. The numerical implementation is done by approximating the irrational square root function by a rational function (Taylor expansion), which is valid for small values of $s$.

On the second order, the general form of E.M. A.B.C. is:

$-\frac{\partial \psi}{\partial \mathrm{n}}=\alpha \psi+\beta \frac{\partial^{2} \psi}{\partial \tau^{2}}+O\left(s^{4}\right)$

where $\alpha=\mathrm{jk}$ and $\beta=\frac{\mathrm{j}}{2 \mathrm{k}}, \mathrm{s}=\frac{\mathrm{k} \tau}{\mathrm{k}}$ (incidence angle)

The primary error associated with this condition is in the approximation applied for the square root, which is only exact for a wave incident normally on the outer boundary (Fig. 2). When the directions of propagation of the waves $\left(\theta_{1}\right.$ and $\theta_{2}$ for example) are known a priori, the condition introduced by Higdon [11] leads to better results. This condition may be seen as a generalization of the E.M. A.B.C., and only the coefficients $\alpha$ and $\beta$ are changed in (10): $\alpha=j k \frac{1+\cos \theta_{1} \cos \theta_{2}}{\cos \theta_{1}+\cos \theta_{2}}$ and $\beta=\frac{j}{k} \frac{1}{\cos \theta_{1}+\cos \theta_{2}}$

\section{B. $2 D$ vector Engquist-Majda A.B.C.}

The same development is made as for the vector B.T. A.B.C.: because each cartesian coordinate of the vector wave A verifies the scalar Helmoltz equation, it may be approximated by the scalar conditon (10); this is also true for the tangential part of $\mathbf{A}$. Using then vector identities, we have the vector A.B.C.:

$\mathbf{n} \times \nabla \times \mathbf{A}=\alpha \mathbf{A}_{\mathbf{t}}+\beta \nabla_{\mathrm{t}}\left(\nabla \cdot \mathbf{A}_{\mathrm{t}}\right)+\nabla_{\mathrm{t}}(\mathbf{n} \cdot \mathbf{A})+\mathrm{O}\left(\mathrm{s}^{4}\right)$

where $\alpha$ and $\beta$ are the same as in (10) or (11)

\section{C. $2 D$ symmetric vector Engquist-Majda A.B.C.}

We use first the same method as the one exposed in [8] for the B.T. A.B.C. (see section II.C). By multipling the vector wave equation by the unit normal vector $\mathbf{n}$, and using the condition (12), we get the following approximation for the normal component of $\mathbf{A}$ :

$-\mathrm{k}^{2} \mathrm{An}_{\mathrm{n}}=\alpha \nabla \cdot \mathbf{A}_{\mathrm{t}}+\nabla_{\mathrm{t}}{ }^{2} \mathrm{An}_{\mathrm{n}}+\beta \nabla_{\mathrm{t}}{ }^{3} \mathbf{A}_{\mathrm{t}}+\mathrm{O}\left(\mathrm{s}^{5}\right)$

On an other hand, the scalar condition (10) is also verified by $\mathrm{A}_{\mathrm{n}}$. Combining it with the free divergence condition leads to:

$-\alpha A_{n}=-\nabla \cdot A_{t}+\beta \nabla_{t}^{2} A_{n}+O\left(s^{4}\right)$

By eliminating the $\nabla \mathrm{t}^{2} \mathrm{An}$ term between (13) and (14), we get an approximation for $\mathbf{n}$.A which leads to the symmetric condition:

$\mathbf{n} \times \nabla \times \mathbf{A}=\alpha \mathbf{A}_{\mathbf{t}}+\beta^{\prime} \nabla_{\mathrm{t}}\left(\nabla . \mathbf{A}_{\mathrm{t}}\right)+\mathrm{O}\left(\mathrm{s}^{4}\right)$

where $\alpha=\mathrm{jk}$ and $\beta^{\prime}=-\frac{\mathrm{j}}{2 \mathrm{k}}$

\section{E. Remark}

When the radius $\mathrm{R}$ of the spherical outer boundary becomes infinite in the expressions of the $2 \mathrm{D}$ vector BaylissTurkel A.B.C. (6) and (8), one finds again the $2 \mathrm{D}$ vector Engquist-Majda A.B.C. (12) and (15). Of course, this remains formal, because $\mathrm{R}$ has to be of finite dimension.

\section{F. $2 D$ corner condition.}

Because a rectangular boundary is no more smooth, a special condition has to be specifically written at the corners of this rectangle to avoid spurious reflections. It is proposed in [6] to combine the scalar wave equation (16) and the absorbing boundary conditions (17.a) and (17.b), derivated from (10), written on both sides $\Gamma x$ and $\Gamma y$ of the corner: 
scalar wave equation: $\frac{\partial^{2} \mathrm{u}}{\partial \mathrm{x}^{2}}+\frac{\partial^{2} \mathrm{u}}{\partial \mathrm{y}^{2}}+\mathrm{k}^{2} \mathrm{u}=0$

boundary $\Gamma \mathrm{x}:-\frac{\partial \mathrm{u}}{\partial \mathrm{y}}+\mathrm{jku}+\frac{\mathrm{j}}{2 \mathrm{k}} \frac{\partial^{2} \mathrm{u}}{\partial \mathrm{x}^{2}}=0$

boundary $\Gamma_{\mathrm{y}}:-\frac{\partial \mathrm{u}}{\partial \mathrm{x}}+\mathrm{jku}+\frac{\mathrm{j}}{2 \mathrm{k}} \frac{\partial^{2} \mathrm{u}}{\partial \mathrm{y}^{2}}=0$

Adding both conditions (17.a) and (17.b) and substracting (16) with the coefficient $\mathrm{j} / 2 \mathrm{k}$ lead to the corner condition (18). This condition may be compared with the one proposed by Engquist and Majda [2], obtained from very different considerations, where $\gamma=\sqrt{2}$.

$\gamma \mathrm{jku}=\frac{\partial \mathrm{u}}{\partial \mathrm{x}}+\frac{\partial \mathrm{u}}{\partial \mathrm{y}}$, with $\gamma=3 / 2$

The vector corner condition is obtained by the same way. Using also the property of $\mathbf{A}$ to be divergence free, we get the condition (19), where $\mathbf{n}$ is the unit vector normal to the line attached to the corner (Fig. 3). An example of implementation of this condition into a F.E. formulation is presented in section IV E.

$\nabla \cdot \mathbf{A t}_{\mathrm{t}}=-\frac{\partial \mathrm{An}}{\partial \mathrm{n}}=\gamma \mathrm{jkAn}$, with $\gamma=\sqrt{2}$ or $3 / 2$

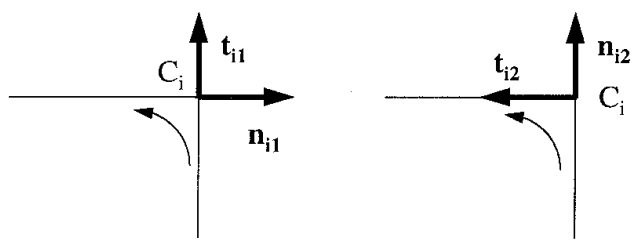

Fig. 3: discontinuity of the unit vectors $\mathbf{n}$ and $\mathbf{t}$ at the corner

G. Example of implementation of the $2 D$ scalar E.M. A.B.C. into the F.E. formulation.

A general formulation for $2 \mathrm{D}$ scattering problems may be found in [10]. It is written for the magnetic field $\mathrm{H}$ :

$$
\int_{S}\left(\nabla N \cdot \frac{1}{j \omega \varepsilon} \nabla H+j \omega \mu N . H\right) d s-\int_{\Gamma} N \cdot \frac{1}{j \omega \varepsilon} \frac{\partial H}{\partial n} d \Gamma=\ldots
$$

The implementation is performed through the line integral. By inserting (10) into (20), this line integral becomes:

$$
-\frac{\mathrm{k}}{\omega \varepsilon} \int_{\Gamma} \mathrm{N} \cdot \mathrm{Hd} \Gamma-\frac{1}{2 \mathrm{k} \omega \varepsilon} \int_{\Gamma} \mathrm{N} \cdot \frac{\partial^{2} \mathrm{H}}{\partial \tau^{2}} \mathrm{~d} \Gamma
$$

The second term of $(21)$ is then integrated by parts. The $\mathrm{N} . \frac{\partial \mathrm{H}}{\partial \tau}$ term coming from this integration is null everywhere, except on the corners. Using then the corner condition (18), the final expression of (21) becomes:

$$
-\frac{\mathrm{k}}{\omega \varepsilon} \int_{\Gamma} \mathrm{N} . \mathrm{H} \mathrm{d} \Gamma+\frac{1}{2 \mathrm{k} \omega \varepsilon} \int_{\Gamma} \frac{\partial \mathrm{N}}{\partial \tau} \cdot \frac{\partial \mathrm{H}}{\partial \tau} \mathrm{d} \Gamma-\frac{3 \mathrm{j}}{4 \omega \varepsilon} \sum_{4 \text { corn. }} \mathrm{N} .\left.\mathrm{H}\right|_{\text {corn. }}
$$

\section{H. Numerical example.}

Fig. 4 shows the error due to the scalar Engquist-Majda A.B.C. in relation with the incidence angle, with and without corner condition (C.C.). We study a free space region, without scatterer. The F.E. domain is a $1.5 \lambda$ side rectangle, and the mesh density is about 18 nodes per wavelength. The global error over the domain is calculated in the same way as in the previous example.

The corner condition divides the global error almost by 2 . As theoritically expected from (10), the error depends on the incidence angle. But the results remain acceptable, even for a $90^{\circ}$ incidence angle (the error is less than $1.5 \%$ ).

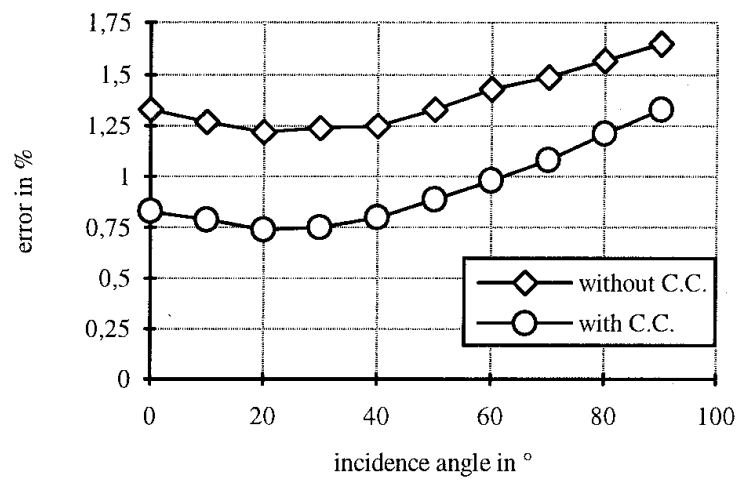

Fig. 4: global error as a function of the incidence angle E.M. A.B.C., with and without corner condition (C.C.) free-space region.

\section{3D ENGQUIST-MAJDA A.B.C}

\section{A. 3D scalar Engquist-Majda A.B.C.}

3D E.M. A.B.C. has been first presented in [13]. It is a natural extension of the two-dimensional one $(10)$ :

$-\frac{\partial \psi}{\partial \mathrm{n}}=\alpha \psi+\beta \nabla_{\mathrm{t}}^{2} \psi+\mathrm{O}\left(\mathrm{s}^{4}\right)$

where $\alpha$ and $\beta$ are the same as in (10)

\section{B. $3 D$ vector Engquist-Majda A.B.C.}

The extension of the scalar case to the vector case is led in the same way as for the 2D case. Each component of the vector wave is solution of the scalar wave equation. Thus, the tangential part of the vector field satisfies (23): 
$-\frac{\partial \mathbf{A} t}{\partial \mathbf{n}}=\alpha \mathbf{A t}+\beta \nabla^{2} \mathbf{A} \mathrm{t}+\mathrm{O}\left(\mathrm{s}^{4}\right)$

A similar expression to the $2 \mathrm{D}$ one (12) is then obtained:

$\mathbf{n} \times \nabla \times \mathbf{A}=\alpha \mathbf{A t}_{\mathrm{t}}+\beta \nabla_{\mathrm{t}}{ }^{2} \mathbf{A}_{\mathrm{t}}+\nabla_{\mathrm{t}}(\mathbf{n} \cdot \mathbf{A})+\mathrm{O}\left(\mathrm{s}^{4}\right)$,

where $\alpha$ and $\beta$ are the same as in (12)

Note that, when developing the $\nabla_{\mathrm{t}}{ }^{2}$ term, (25) leads to the non-symmetric vector A.B.C. proposed by Peterson in [14]:

$$
\begin{aligned}
\mathbf{n} \times \nabla \times \mathbf{A}= & \alpha \mathbf{A}_{\mathrm{t}}-\beta \nabla \times\{\mathbf{n} \cdot(\nabla \times \mathbf{A})\} \mathbf{n}+\beta \nabla_{\mathrm{t}}\left(\nabla \cdot \mathbf{A}_{\mathrm{t}}\right) \\
& +\nabla_{\mathrm{t}}(\mathbf{n} \cdot \mathbf{A})+\mathrm{O}\left(\mathrm{s}^{4}\right),
\end{aligned}
$$

\section{3D symmetric vector Engquist-Majda A.B.C.}

Once more, due to the last term $\nabla \mathrm{t}$, the implementation of (25) into a F.E. formulation leads to a non-symmetric system matrix. Proceeding as previously leads to the symmetric condition:

$\mathbf{n} \times \nabla \times \mathbf{A}=\alpha \mathbf{A}_{\mathrm{t}}+\beta \nabla_{\mathrm{t}}^{2} \mathbf{A}_{\mathrm{t}}+\beta^{\prime \prime} \nabla_{\mathrm{t}}\left(\nabla \cdot \boldsymbol{A}_{\mathrm{t}}\right)+\mathrm{O}\left(\mathrm{s}^{4}\right)$

where $\alpha=j k, \beta=\frac{j}{2 k}$ and $\beta^{\prime \prime}=-\frac{j}{k}$

Again, when the $\nabla_{\mathrm{t}}^{2}$ term is developed, one identifies the shape of the second order symetric conditions introduced in (8).

\section{D. $3 D$ edge and corner condition}

Let's consider the edge $\mathrm{L}_{\mathrm{ij}}$, intersection of the rectangular outer surfaces $\Gamma_{i}$ and $\Gamma_{j}$ (Fig. 5). From (24), we can write the absorbing boundary condition on the edge $L_{i j}$ for both surfaces $\Gamma_{\mathrm{i}}$ and $\Gamma_{\mathrm{j}}$ :

$\mathrm{Lij}_{i \mathrm{i}} \in \Gamma_{\mathrm{i}}:-\frac{\partial \mathrm{Al}}{\partial \mathrm{n}_{\mathrm{j}}}=\alpha \mathrm{Al}+\beta\left(\frac{\partial^{2} \mathrm{Al}}{\partial \mathrm{l}^{2}}+\frac{\partial^{2} \mathrm{Al}}{\partial \mathrm{ni}^{2}}\right)$

$\mathrm{Lij}_{\mathrm{ij}} \in \Gamma_{\mathrm{j}}:-\frac{\partial \mathrm{Al}}{\partial \mathrm{ni}_{\mathrm{i}}}=\alpha \mathrm{Al}+\beta\left(\frac{\partial^{2} \mathrm{Al}}{\partial \mathrm{l}^{2}}+\frac{\partial^{2} \mathrm{Al}}{\partial \mathrm{n}^{2}}\right)$

Combining both of these conditions with the vector wave equation leads to the corner condition:

on $\mathrm{Lij}_{\mathrm{ij}}:-\left(\frac{\partial \mathrm{Al}}{\partial \mathrm{ni}_{\mathrm{i}}}+\frac{\partial \mathrm{Al}}{\partial \mathrm{n}_{\mathrm{j}}}\right)=\left(2 \alpha-\mathrm{k}^{2} \beta\right) \mathrm{Al}+\beta \frac{\partial^{2} \mathrm{Al}}{\partial 1^{2}}$

Because of the respective values of $\alpha$ and $\beta$, the final expression of the corner condition (29) is:

on $\mathrm{Lij}_{\mathrm{ij}}:-\left(\frac{\partial \mathrm{Al}_{\mathrm{l}}}{\partial \mathrm{ni}_{\mathrm{i}}}+\frac{\partial \mathrm{Al}}{\partial \mathrm{n}_{\mathrm{j}}}\right)=\frac{3 \mathrm{jk}}{2} \mathrm{~A} \mathrm{l}+\frac{\mathrm{j}}{2 \mathrm{k}} \frac{\partial^{2} \mathrm{Al}}{\partial \mathrm{l}^{2}}$

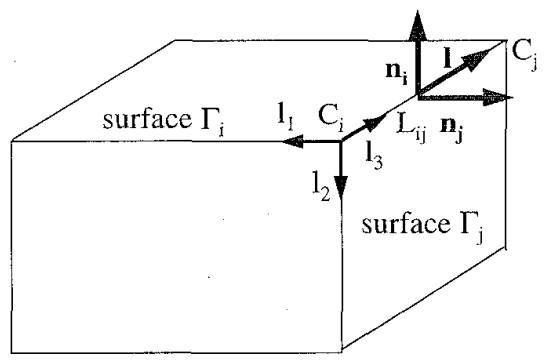

Fig. 5: definition of edge and corner on the $3 \mathrm{D}$ rectangular outer boundary.

The corner condition in 3D is formally the same as the $2 \mathrm{D}$ one (19). The sign + or - depends on the orientation of the tangent vector $l$ on the corner. We have then:

$-\frac{\partial \mathrm{Al}}{\partial \mathrm{l}}= \pm \gamma \mathrm{jkAl}$

E. Example of implementation of the $3 D$ vector E.M. A.B.C. into the F.E. formulation.

In [15], we presented a 3D vector F.E. formulation:

$$
\begin{aligned}
\int_{\Omega}\left[\frac{1}{\varepsilon_{\mathrm{r}}}(\nabla \times \mathbf{H}) \cdot(\nabla \times \mathbf{W})-\mathrm{k}_{0}{ }^{2} \mu \mathrm{H} \cdot \mathbf{W}\right] \mathrm{d} \Omega \\
+\int_{\Gamma}(\mathbf{n} \times \nabla \times \mathbf{H}) \cdot \mathbf{W} \mathrm{d} \Gamma=\ldots
\end{aligned}
$$

The vector E.M. condition (27) is used to approximate the surface integral term. After an integration by parts, this surface term becomes:

$\mathrm{jk} \int_{\Gamma} \mathbf{H}_{\mathrm{t}}, \mathbf{W}_{\mathrm{t}} \mathrm{d} \Gamma-\frac{\mathrm{j}}{2 \mathrm{k}} \int_{\Gamma}\left(\frac{\partial \mathbf{H}_{\mathrm{t}}}{\partial \tau} \cdot \frac{\partial \mathbf{W}_{\mathrm{t}}}{\partial \tau}+\frac{\partial \mathbf{H}_{\mathrm{t}}}{\partial \mathrm{v}} \cdot \frac{\partial \mathbf{W}_{\mathrm{t}}}{\partial \mathrm{v}}\right) \mathrm{d} \Gamma$

$+\frac{\mathrm{j}}{\mathrm{k}} \int_{\Gamma}\left(\nabla \cdot \mathbf{H}_{\mathrm{t}}\right)(\nabla \cdot \mathbf{W} \mathbf{t}) \mathrm{d} \Gamma$

$+\frac{j}{2 \mathrm{k}} \sum_{\mathrm{i}=1}^{6} \oint_{\partial \Gamma \mathbf{i}} \frac{\partial \mathbf{H}_{\mathrm{t}}}{\partial \mathrm{n}_{\mathrm{i}}} \cdot \mathbf{W}_{\mathrm{t}} \mathrm{dl}-\frac{\mathrm{j}}{\mathrm{k}} \sum_{\mathrm{i}=1}^{6} \oint_{\partial \Gamma \mathrm{i}}\left(\nabla . \mathbf{H}_{\mathbf{t}}\right)\left(\mathbf{n} \mathbf{i} \cdot \mathbf{W}_{\mathbf{t}}\right) \mathrm{dl}$

Edge (30) and corner (31) conditions are used to evaluate the last two terms of (33). The final symmetric expression of the surface term in (32) is then:

$j \mathrm{k} \int_{\Gamma} \mathbf{H}_{\mathrm{t}} \cdot \mathbf{W}_{\mathrm{t}} \mathrm{d} \Gamma-\frac{\mathrm{j}}{2 \mathrm{k}} \int_{\Gamma}\left(\frac{\partial \mathbf{H}_{t}}{\partial \tau} \cdot \frac{\partial \mathbf{W}_{\mathrm{t}}}{\partial \tau}+\frac{\partial \mathbf{H}_{t}}{\partial \mathrm{v}} \cdot \frac{\partial \mathbf{W}_{\mathrm{t}}}{\partial \mathrm{v}}\right) \mathrm{d} \Gamma$

$+\frac{\mathrm{j}}{\mathrm{k}} \int_{\Gamma}\left(\nabla \cdot \mathbf{H}_{\mathrm{t}}\right)\left(\nabla \cdot \mathbf{W}_{\mathrm{t}}\right) \mathrm{d} \Gamma+\sum_{\mathrm{i}<\mathrm{j}} \int_{\mathrm{Lij}}\left(\mathrm{H}_{\mathrm{ni}} \mathrm{W}_{\mathrm{ni}}+\mathrm{H}_{\mathrm{nj}} \mathrm{W}_{\mathrm{nj}}\right) \mathrm{dl}$

$-\frac{j}{2 k} \sum_{i<j} \int_{L i j j}\left( \pm j k H_{l} W_{l}+\gamma j k H_{n i} W_{n}+\gamma j k H_{n j} W_{n j}-\frac{j}{2 k} \frac{\partial H_{l}}{\partial l} \cdot \frac{\partial W_{l}}{\partial l}\right) d l$

$+\left.\frac{r j}{4 k} \sum_{k=1}^{8}\left( \pm \mathrm{H}_{11} \mathrm{~W}_{11} \pm \mathrm{H}_{12} \mathrm{~W}_{12} \pm \mathrm{H}_{13} \mathrm{~W}_{13}\right)\right|_{\mathrm{Ck}}$ 
From (34), it is obvious that taking into account the geometric singularities makes the implementation of the A.B.C. more complex. In first approximation, edge and corner conditions could be omitted. By doing this, we assume that the spurious waves generated by these singularities are essentially local, and that only a small amount of them propagates toward the interior of the domain. Next exemple will justify this approximation.

\section{F. Numerical example.}

We compare the 3D scalar E.M. A.B.C., as it is implemented in nodal F.E. formulation [15], with the vector E.M. A.B.C., implemented in mixed F.E. formulation [16], for the radiation by an infinitesimal dipole (fig. 6). The dipole, with a constant current, is centered on the origin and directed along the $z$-axis. The problem has been modeled with two symmetries ( $x z$ and $y z$ planes). The size of the computational domain is $1 \lambda \times 1 \lambda \times 2.1 \lambda$. Each finite element is a brick $0.1 \lambda \times 0.1 \lambda \times 0.1 \lambda$.

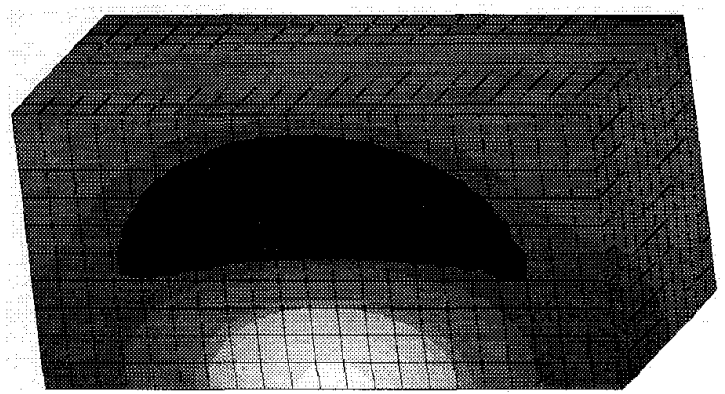

Fig. 6: instantaneous $\mathbf{H}$ radiated by an infinitesimal dipole plot on the symmetry planes and on the outer surfaces.

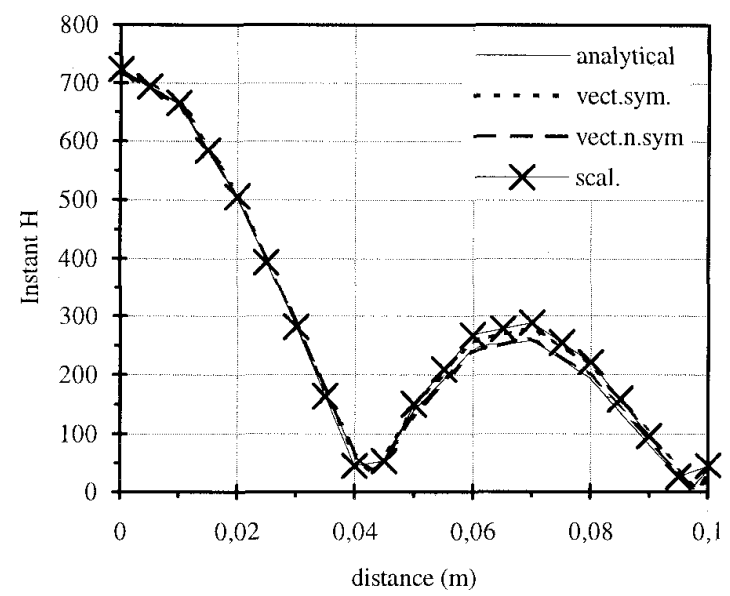

Fig. 7: instantaneous $\mathbf{H}$ along a line perpendicular to the dipole, going from the dipole to the external boundary.

Fig. 7 shows analytical and numerical solutions plotted on a vertical line, going from the dipole to the external boundary. In the interior of the domain, the solutions are quite close.
The accuracy of the non-symmetric A.B.C. is approximately the same as the symmetric one.

\section{CONCLUSION}

We have presented in this paper two types of absorbing boundary conditions: the Bayliss-Turkel A.B.C., for spherical outer boundary, and the Engquist-Majda A.B.C., for rectangular outer boundary. We have shown how it is possible to symmetrize them with the same order of precision as the underlying non-symmetric conditions. Edge and corners conditions for the E.M. A.B.C. have also been presented. If these special conditions are easy to implement in $2 \mathrm{D}$, the $3 \mathrm{D}$ formulation becomes much more complex, and they can be omitted without notable damaging of the solution.

\section{REFERENCES}

[1] D. Givoli, "Non-reflecting boundary conditions," J. Comput. Phys, vol. 99, pp. 1-29, 1991.

[2] B. Engquist, A. Majda, "Radiation boundary conditions for acoustic and elastic wave calculations," Comm. Pure Appl., vol. 32, pp. 313-357, 1979.

[3] A. Bayliss, E. Turkel, "Radiation boundary conditions for wave-like equations," Comm. Pure Appl. Math., vol. 33, pp. 707-725, 1980.

[4] S.N. Karp, "A convergent far-field expansion for two-dimensional radiation functions," Comm. Pure Appl. Math., vol. 32, pp. 313-357, 1979.

[5] R. Mittra, O. Ramahi, "Absorbing boundary conditions for the direct solution of partial differential equations arising in electromagnetic scattering problems," in Finite Element and Finite Difference Methods in Electromagnetic Scattering, vol. II, M.A. Morgan Ed. New York: Elsevier, 1990, pp. 133-173.

[6] J.L. Yao Bi, L. Nicolas, A. Nicolas, "Modeling unbounded wave propagation in terms of transverse fields using $2 \mathrm{D}$ mixed finite elements," IEEE Trans. on Mag., vol. 31, no 3, pp. 1594-1597, may 1995.

[7] J.L. Yao Bi, "Méthode des élements finis mixtes et C.A.L. absorbantes pour la modélisation des phénomènes électromagnétiques hyperfréquences," Ph.D. thesis, E.C.L., 1995.

[8] A.F. Peterson, "Accuracy of 3D radiation boundary conditions for use with the vector Helmoltz equation", IEEE Trans. Antennas Propag., vol. 40, no. 3, pp 351-355, 1992.

[9] C.A. Balanis, Advanced engineering electromagnetics, John Wiley \& Sons New York, 1989.

[10] J.L. Yao Bi, L. Nicolas, A. Nicolas, "2D electromagnetic scattering by simple shapes: a quantification of the error due to open boundary," IEEE Trans. on Mag., vol. 29, no 2, pp. 1830-1834, 1993.

[11] R.L. Higdon, "Numerical absorbing boundary conditions for the wave equation", Math. Comp.., vol 49, pp 65-90, 1987.

[12] A. Bamberger, P. Joly, J.E. Roberts, "Second order absorbing boundary conditions for the wave equation: a solution for the corner problem",SIAM J. Numer. Anal., vol 27, no 2, pp 323-352, 1990.

[13] J. D'Angelo, I.D. Mayergoyz, "On the use of local absorbing boundary conditions for RF scattering problems," IEEE Trans. on Mag., vol. 27, no 5, pp.3827-3832, 1991.

[14] A.F. Peterson, "Absorbing boundary conditions for theVector wave equation", Microwave and Optical Technology Letters, vol. 1, no. 2, pp. 62-64, 1988.

[15] L. Nicolas, K.A. Connor, S.J. Salon, B.G. Ruth, L.F. Libelo, "Three dimensional finite element analysis of high power microwave devices," IEEE Trans. on Mag., vol. 29, pp. 1642-1645, march 1993.

[16] J.L. Yao Bi, L. Nicolas, A. Nicolas, "H(curl) elements on hexahedral and vector A.B.C.'s for unbounded microwave problems," IEEE Trans. on Mag., vol. 31, no 3, pp. 1538-1541, may 1995. 